\title{
CORDE VIBRANTE AVEC AMORTISSEMENTS, DANS LE FORMALISME D'IMPÉDANCE
}

\author{
C. VALETTE
}

Laboratoire d'Acoustique Musicale, Université Paris 6, CNRS URA $N^{\circ} 868, T .66$, Sème ét., 4 pl. Jussieu, F-75252 Paris cedex 05, France

\begin{abstract}
The string vibration is modified by various linear perturbations, especially by damping arising from viscosity, viscoelasticity and thermal transport. The complex pulsation is computed to first order, dispersion and absorbtion effects are discussed.
\end{abstract}

Dans un cadre de théorie modale, le mouvement du chevalet et les différents mécanismes d'amortissement, plus ou moins nombreux et compliqués dans la pratique [1] (effets de viscosité, de transport thermique etc...), apporte une perturbation linéaire. Les phénomènes de couplage de modes (dus aux non-linéarités [*]) étant négligés, on traite chaque mode séparément. La viscoélasticité provoque un retard de la déformation sur la contrainte. L'effet thermoélastique nécessite [2] une moyenne géométrique dans le fil. Ces effets, calculés en annexe, font apparaître un angle de perte $\delta=\delta_{v e}+\delta_{t e}$ sur le module d'Young. Dans le cas de fils métalliques, les dislocations [3] interviennent dans ces deux mécanismes. D'autres termes de pertes peuvent être introduits pour décrire des mécanismes plus compliqués présents dans des cordes non monofilament. Nous exprimons le nombre d'onde $k$ en fonction de l'admittance au chevalet puis la pulsation complexe $\omega$ à partir de la relation de dispersion.

\section{I- ONDES TRANSVERSALES DANS UNE CORDE MONOFILAMENT}

Pour un mouvement transversal plan, la vitesse $u(x, t)$ et l'effort tranchant $f(x, t)$ sont reliés dans l'approximation des petits déplacements et des petits angles par:

$\dot{f}=-T u^{\prime}+E(1-j \delta) I u^{\prime \prime \prime}, \quad f^{\prime}=-\rho i \dot{u}-2 r u$

où $2 r$ est une résistance de frottement visqueux (air par exemple).

Avec des conditions aux limites d'appui simple au sillet (en $x=0$, supposé immobile) et au chevalet (en $x=L$, supposé d'admittance $Y_{c h}$ petite), $u$ est de la forme $u(x, t)=u_{0} \sin k x \exp -j \omega t$

et $k$ s'écrit au premier ordre (voir (5)):

$k=\frac{n \pi}{L}\left\{1-j \frac{Z_{c} Y_{c h}}{\pi}(n)^{-1}\right\}$, avec $Z_{c}=\left(\rho_{l} T\right)^{1 / 2}$

Dès que $Y_{c h}$ a une partie réelle (transmission d'énergie), $k$ devient complexe. 
Corde de faible raideur fortement précontrainte (usage musical)

La relation de dispersion s'écrit (dispersion supposée faible)

$\omega=c k\left\{1+\frac{1}{2} \frac{E(1-j \delta) I}{T} k^{2}-j \frac{r}{\rho_{l}}(\omega)^{-1}\right\}, \quad$ avec $c=\left(\frac{T}{\rho_{l}}\right)^{1 / 2}$

et on obtient pour $\omega$, toujours au premier ordre :

$\omega=\frac{n \pi c}{L}\left\{1+\frac{2 \pi^{2} \rho E(1-j \delta) I}{T^{2}}\left(n f_{o}\right)^{2}-j \frac{r}{2 \pi \rho_{l}}\left(n f_{o}\right)^{-1}-j \frac{Z_{c} Y_{c h}}{\pi}(n)^{-1}\right\}$, avec $f_{o}=\frac{c}{2 L}$

Précisons que le calcul est valable tant qu'on peut trouver $\varepsilon$, définissant l'infiniment petit du premier ordre, tel que:

$\frac{2 \pi^{2} \rho E I}{T^{2}}\left(n f_{o}\right)^{2}<\varepsilon, \frac{Z_{c}\left|Y_{c h}\right|}{\pi}(n)^{-1}<\varepsilon, \frac{r}{2 \pi \rho_{l}}\left(n f_{o}\right)^{-1}<\varepsilon, \varepsilon \ll 1$

Par rapport à la corde idéale, la fréquence est modifiée principalement par l'inharmonicité de raideur et la partie imaginaire de l'admittance au chevalet, ce dernier effet pouvant partiellement compenser le premier (correction due à la résistance de frottement négligeable):

$f=n f_{o}\left\{1+\frac{2 \pi^{2} \rho E I}{T^{2}}\left(n f_{o}\right)^{2}+\frac{Z_{c} I_{m} Y_{c h}}{\pi}(n)^{-1}\right\}$

Les amortissements et la transmission d'énergie au chevalet entraînent une décroissance de l'amplitude dans le temps, représentée par le facteur de qualité:

$Q^{-1}=\frac{r}{\pi \rho_{l}}\left(n f_{o}\right)^{-1}+\frac{4 \pi^{2} \rho_{l} E I}{T^{2}}\left(\delta_{v e}+\delta_{t e}\right)\left(n f_{o}\right)^{2}+\frac{2 Z_{c} R_{l} Y_{c h}}{\pi}(n)^{-1}$

Le pourcentage d'énergie transmise au chevalet est fonction du rapport entre l'impédance caractéristique de la corde et l'impédance d'entrée au chevalet. Les pertes dans l'air dominent a basse fréquence, les partiels de rang élevé sont fortement amortis par la viscoélasticité. Les fréquences intermédiaires sont favorisées et $\boldsymbol{Q}$ a un maximum qui, pour les cordes utilisées musicalement, tombe dans la zone sensible de l'oreille.

L'effet thermoélastique dépend de la conductivité thermique, sensible aux dislocations. $\Pi$ reste masqué par les frottements dans l'air et l'effet viscoélastique, sauf au voisinage du maximum de $Q$. Pour des cordes typiques de clavecin en laiton, il semble [3] que la fréquence de résonnance soit peu inférieure à celle du maximum de $Q$ quand la corde est peu écrouie à la fabrication. La première mise en tension entraîne un écrouissage, donc une diminution de la conductivité thermique, ce qui entraîne une diminution des pertes thermoélastiques : on dit que "la corde se fait".

Verge isolée non précontrainte

La relation de dispersion devient quadratique, d'où l'expression de $\omega$ et de $Q$ : $\omega=\left(E I / \rho_{l}\right)^{1 / 2}\left(\frac{n \pi}{L}\right)^{2}\left(1-j \frac{\delta_{v e}+\delta_{t e}}{2}\right)$, et $Q^{-1}=\delta_{v e}+\delta_{t e}$

Le facteur de qualité ne dépend plus de la fréquence, contrairement à [8].

\section{II- GÉNÉRALISATION： AUTRES MOUVEMENTS, AUTRES TYPES DE CORDES}

\section{Ondes longitudinales, ondes de torsion}

La vitesse de propagation est affectée par l'effet viscoélastique, l'effet thermoélastique est négligeable (voir (22)) dans le premier cas, nul dans le second: $Q^{-1}=\delta_{v e}$ 


\section{Cordes non monofilament}

Les mesures effectuées au Laboratoire sur diverses cordes non monofilament [4] montrent l'existence d'autres mécanismes de perte plus compliqués. Par exemple, certaines cordes en boyaux commis, ainsi que les cordes en fibre végétale de la valiha (instrument joué à Madagascar), ont des frottements de type visqueux à basse fréquence autres que celui de l'air. Dans certaines cordes filées, des frottements entre spires peuvent introduire une forte absorbtion à moyenne fréquence. L'introduction d'un angle de perte sur $T$ dans (1), analogue formel du classique effet de transport thermique dans la couche limite des tuyaux sonores, pourrait décrire des frottements relatifs à l'effort tranchant. D'autres termes de perte pourraient être rajoutés.

\section{CONCLUSION}

La pulsation complexe décrit dans sa partie imaginaire les effets d'amortissement, et dans sa partie réelle ceux de dispersion. Contrairement aux instruments à vent, la diffusion liée à la viscosité ou au transport thermique n'affecte pas la fréquence sensiblement. Formellement, tout terme d'amortissement peut être écrit sous forme d'une résistance de frottement visqueux, fonction de la fréquence. Mais si l'on veut garder évident le sens physique lié au mécanisme, l'utilisation de termes séparés dans les équations de départ du formalisme d'impédance (1) est plus claire.

Nous remercions tout particulièrement le Ministère de la Culture pour l'aide soutenue qu'il apporte à notre laboratoire.

\section{ANNEXES}

\section{A.I- Angle de perte sur $\mathrm{E}$, dû aux effets viscoélastiques}

La déformation se produit avec un léger retard sur la contrainte car elle nécessite, pour les métaux, le glissement des dislocations : ce sont les points d'ancrage (défauts ponctuels) des dislocations qui glissent de site en site. Cet effet, responsable de la défaillance du modèle de Granato et Lücke pour les fréquences inférieures à $30 \mathrm{kHz}$, ne dépend pas de la fréquence [3]. II en résulte un angle de perte $\delta_{v e}$ petit et indépendant de la fréquence (correction sur la partie réelle négligeable).

\section{A-II-Amortissement thermoélastique en flexion}

Nous supposons que la corde a la forme d'un ruban de largeur $l$, d'épaisseur $d$, et nous repérons par la coordonnée $z$ un point dans l'épaisseur du ruban. En suivant Zener [2], nous introduisons un mode propre transversal et définissons la température locale :

$y(x, t)=\mathcal{Y}(x) \exp -j \omega t, \quad \Theta(z, t)=\theta_{0}+\theta(z, t)$

L'échauffement est proportionnel à la déformation. D'autre part la contrainte normale est, du fait de la dilatation, affectée par l'échauffement. On définit les deux coefficients de proportionnalité:

$\beta=(\mathrm{d} \Theta / \mathrm{d} e)_{\text {adiab. }}$ et $\alpha=(\mathrm{dX} / \mathrm{d} \Theta)$ contr.

En introduisant la constante $D$ de diffusion de la chaleur, le bilan thermique conduit, avec des conditions aux limites adiabatiques à la surface de la corde, à :

$\theta(z, t)=\left(z-\frac{\sinh \kappa z}{\kappa \cosh k d / 2}\right) \beta Y_{x}^{\prime \prime} \exp -j \omega t$, avec $\kappa^{2}=-j \frac{\omega}{D}$

On calcule la correction apportée par cet échauffement sur l'effort tranchant, par l'intermédiaire du moment fléchissant. Finalement, l'effet thermoélastique apporte une correction sur le module d'Young qui devient complexe, avec un angle de perte: 
$E\left(1+\frac{\alpha \beta}{E}\right)\left(1-\frac{12 \alpha \beta}{E} \frac{1}{\kappa^{2} d^{2}}\left(1-\frac{2 \tanh \kappa d / 2}{x d}\right)\right), \quad \delta_{t e}=\frac{12 \alpha \beta}{E} \frac{D}{d^{2} \omega} R_{\ell}\left(1-\frac{2 \tanh \kappa d / 2}{x d}\right)$

Lorsque la dimension caractéristique liée à l'équation de diffusion est de l'ordre de l'épaisseur de la corde, il y a résonnance :

$f \approx \frac{\pi D}{4 d^{2}}, \quad k d \approx \frac{\pi}{2}(1-j), \quad \delta_{t e} \approx \frac{0,4 \alpha \beta}{E}$

En dessous de cette fréquence le régime est isotherme, la partie réelle reste inchangée, $E$, et l'angle de perte nul :

$f<\frac{\pi D}{4 d^{2}}, \quad \delta_{i e} \approx 0$

Au dessus de cette fréquence (régime adiabatique), la correction sur la partie réelle reste négligeable, l'angle de perte diminue en 1/f et devient négligeable devant l'angle de perte viscoélastique, qui reste lui constant:

$f>\frac{\pi D}{4 d^{2}}, \quad \delta_{r e} \approx \frac{12 \alpha \beta}{E} \frac{D}{d^{2} \omega}$

Cest donc autour de la fréquence de résonnance que l'effet relatif par rapport à la viscoélasticité est le plus grand.

A-III- Amortissement thermoélastique en compression

L'effet thermoélastique se calcule facilement en suivant une démarche analogue à celle de A-II, avec un mode longitudinal :

$y(x, t)=y_{0} \sin k x \exp -j \omega t$

Le bilan thermique donne (conditions adiabatiques aux extrémités):

$\theta(x, t)=-\beta k\left(1-\left(\frac{k}{k}\right)^{2}\right) y_{0} \cos k x \exp -j \omega t$, avec $\kappa^{2}=-j \frac{\omega}{D}$

Le module d'Young devient complexe, avec un angle de perte thermoélastique, mais l'effet est négligeable car $k / \kappa \ll 1$ :

$E\left(1+\frac{\alpha \beta}{E}\right)\left(1-j \frac{\alpha \beta}{E} \frac{D k^{2}}{\omega}\right), \quad \delta_{t e}=\frac{\alpha \beta}{E} \frac{\rho_{u} D \omega}{E}$

\section{BIBLIOGRAPHIE}

[1] Cuesta C. et Valette C., Evolution temporelle de la vibration des cordes de clavecin, Acustica 43 (1988) 37-45.

[2] Zener C., Internal friction in solids, Phys. Rev. 52 (1938) 230, 53 (1938) 90 et 100.

[3] Simpson H.M. and Sosin A., Contribution of defect dragging to dislocation damping, Phys. Rev. B 5 (1972) 1382-1392.

[4] Besnainou C., Caracciolo A., Cuesta C., Razafindrakoto J., communications privées.

[*] Watzky A., dans le cadre d'un remarquable travail sur la vibration non linéaire des poutres précontraintes a expliqué le mécanisme de génération des modes axiaux à partir des modes transversaux. Sa contribution à ce congrès a été refusée. 